\title{
The research on the Application of Fuzzy Immune PD Algorithm in the Two-Wheeled and Self-Balancing Robot System
}

\author{
Jian Fang \\ Institute of electrical engineering, Jilin Teachers' Institute of Engineering and \\ Technology, Changchun 130052, China \\ $757314739 @ q q . c o m$
}

\begin{abstract}
The fuzzy immune PD controller is designed in view of the nonlinear and model uncertainty characteristics of the two-wheeled and self-balancing robot, and the simulation study is carried out. The simulation results show that the designed control system has the advantages of small overshoot amount, short adjustment time and strong anti-jamming capability compared with the conventional fuzzy PD controller. It is suitable for the balance of the robot control system whose mathematical model is difficult to determine and when the parameter changes.
\end{abstract}

Keywords: fuzzy immune; PD controller; self-balancing robot; mathematical model

\section{Introduction}

The two-wheeled and self-balancing robot is a multivariable, nonlinear and unstable nature controlled object, where the effective balance control has been attracting the attention of scholars, and many kinds of control scheme are proposed. The traditional parameter adjustment of PID control is difficult, and it has the problem of frequent oscillation and large overshoot amount [1]. The optimal LQR controller can correct the two-wheeled balancing robot system in a short period of time [2]. It makes the system reach the equilibrium state and has the good robustness and stability, but it is difficult to determine the weighted matrix $\mathrm{Q}$ and R[3-4]. The above two methods are dependent on the accurate mathematical model of the controlled object and often difficult to obtain the accurate mathematical model of the object in the practical application, so it makes the application scope of PID and LQR controller restricted. In view of the practical problem that it is difficult to model, the two-wheeled and self-balancing robot control system which is based on the fuzzy control can overcome the instability and nonlinear nature of the system, but the fuzzy rules are too much and increases the difficulty of the reasoning[5-6]. This paper combines the fuzzy control and immune PD controller to design the fuzzy immune PD controller. The controller not only inherits the advantages of the traditional fuzzy control, which does not depend on the object mode and has the less fuzzy rules and is easy to project implementation, but also it overcomes the shortcomings of the traditional PD and LQR controllers which are poor in the adjusting follow-up when it is in the strong interference or highly nonlinear nature and uncertainty. Therefore, it improves the performance of the controller greatly. We can see this method has the good static and dynamic performance through the simulation test and comparison, and it is more suitable for the posture balance control of the two-wheeled and self-balancing robot. 
But the immune system fuzzy PI controller design is more complex than the immune PD control and fuzzy control, adjust parameters more.

The control system of self-balancing robot is mainly composed of four parts. The first is the core controller unit, which uses MOTOROLA 32-bit processor; the model is K60 used to analyze the above attitude data information. The corresponding control algorithm calculation is made to make control decisions, and the control signal is sent to the drive unit to control the drive motor; the second is the sensor unit of the robot, which includes the gyroscope, accelerometer and speed sensor [7] used to measure the real-time attitude information of the robot. The third is the robot drive unit, which adjusts the motion state of the two motors according to the signal from the controller; The fourth is robot communications unit, which implements the PC modification parameter and the machine controller saves the receiving parameters in the EEPROM; at the same time, the machine transmits the real-time posture information of the robot to the PC monitoring interface. The control system hardware structure diagram is shown in Figure 1-1.

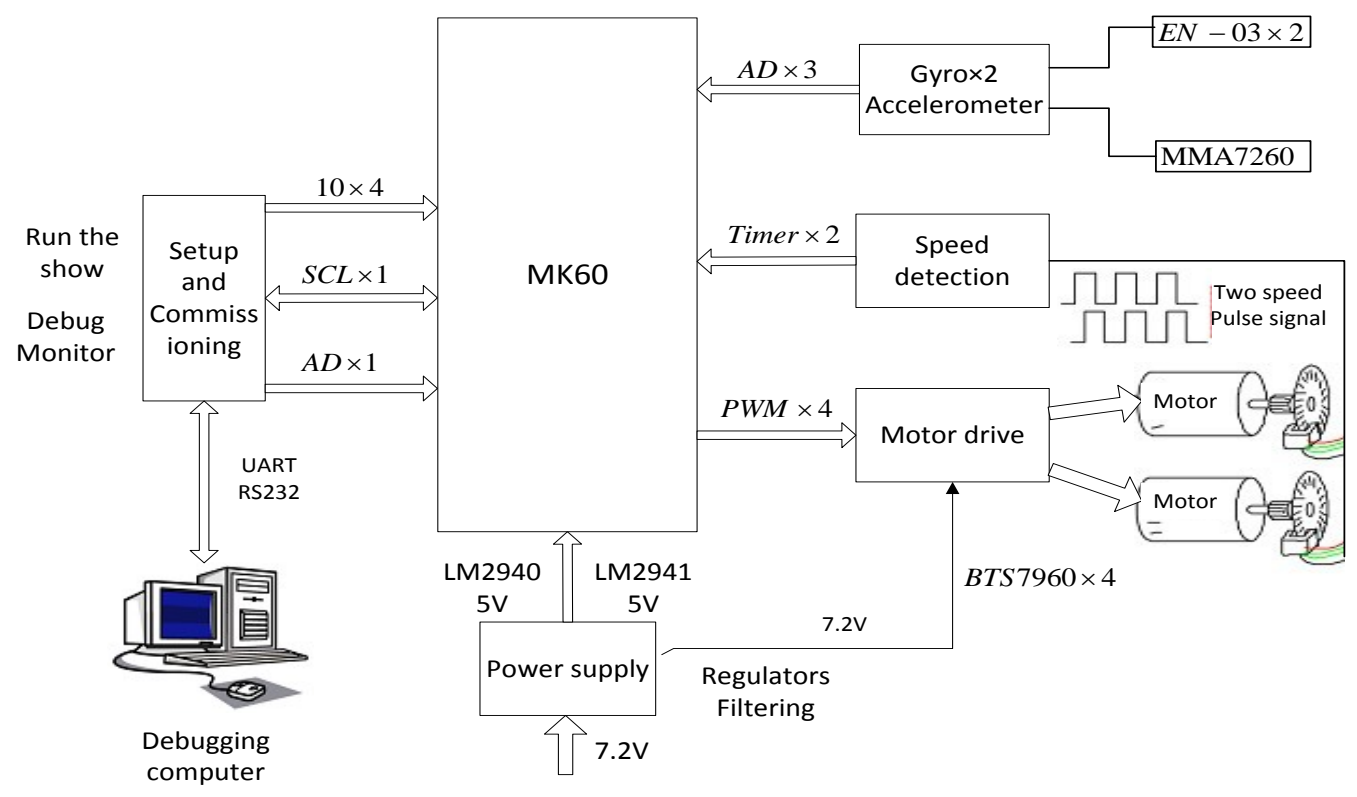

\section{Figure 1-1. The Structure Diagram of the Two-Wheeled Self-Balancing Robot System}

\section{The Posture Control Principle of the Two-wheeled Self-balancing Robot}

The two-wheeled and self-balancing robot can be regarded as the simple inverted pendulum whose high is $\mathrm{L}$ and quality is $\mathrm{m}$; it is placed on the wheel which can move around. As shown in Figure 2-1, if we want to make the inverted pendulum like a pendulum stabilized in the vertical position through the control, there are only two ways: one is to change the direction of gravity, and another is increasing the additional stress to make the restoring force and displacement in the opposite direction. However, it is obvious what we can do is only the second way. 


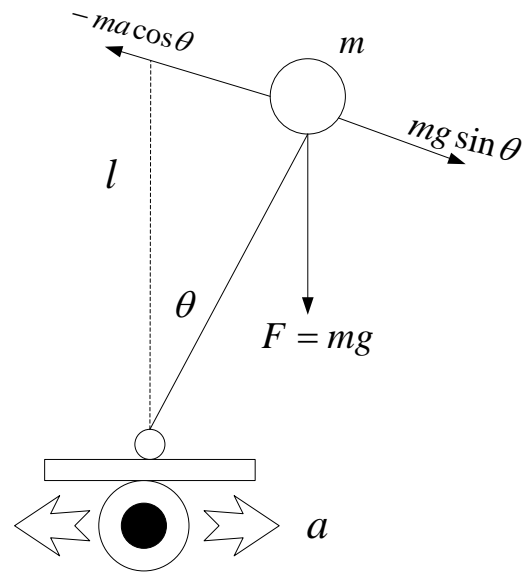

Figure 2-1. The Motion Analysis of the Two-Wheeled Mobile Robot

The wheel at the bottom of the inverted pendulum is controlled to make it do the accelerated motion. If we stand on the mobile robot (non-inertial system) and see the inverted pendulum, it will get an additional force (inertia force); the direction of this force is opposite to the acceleration of the wheel, and the size is in proportion to it. The restoring force which the inverted pendulum receives is:

$$
F=\mathrm{m} g \sin \theta-m a \approx m g \theta-m k_{1} \theta
$$

The formula assumes that the control wheel acceleration is in proportion to the deflection, and the ratio is $\mathrm{k} 1$. Obviously, if $\mathrm{k} 1>\mathrm{g}$ (acceleration of gravity), then the direction of the restoring force is opposite to the displacement. In addition, in order to make the inverted pendulum stabilize in the vertical position as soon as possible, we also need to increase the damping force, which is in proportion to the speed of the deflection, and the direction is opposite. Therefore, Equation (1) can be changed into:

$$
F=\mathrm{m} g \theta-m k_{1} \theta-m k_{2} \theta^{\prime}
$$

Therefore, the control algorithm of the wheel acceleration of the two-wheeled selfbalancing robot is:

$$
\mathrm{a}=k_{1} \theta+k_{2} \theta
$$

However, we need to measure the two-wheeled self-balancing robot angle $\theta$ and angular velocity $\theta^{\prime}$, and it is the controlled up-right, namely the PD control.

\section{Main Title The Fuzzy Immune PD Controller}

\subsection{The Immune PD Control Principle}

The immune PD control algorithm realizes the control function through learning from the immune mechanism in the biological systems [8]. Based on the biological immune feedback principle [10], the immune control algorithm is formulated as follows: let the number of 
antigen of $\mathrm{k}$ is $\varepsilon(k)$, the output of the cells stimulated by antigen is $\mathrm{T}_{\mathrm{H}}(\mathrm{k})$, the influence of Ts cells on B cells is $T_{s}(k)$, and the total stimulus $S(k)$ which the B cells receive is:

$$
\mathrm{S}(\mathrm{k})=\mathrm{T}_{\mathrm{H}}(\mathrm{k})-\mathrm{T}_{\mathrm{s}}(\mathrm{k})
$$

Among them:

$$
\begin{gathered}
\mathrm{T}_{\mathrm{H}}(\mathrm{k})=\mathrm{k}_{1} \varepsilon(\mathrm{k}) \\
\mathrm{T}_{\mathrm{S}}(\mathrm{k})=\mathrm{k}_{2} \mathrm{f}[\mathrm{S}(\mathrm{k}), \quad \Delta \mathrm{S}(\mathrm{k})] \varepsilon(\mathrm{k})
\end{gathered}
$$

In the above formula, $\mathrm{k}_{1}$ is the stimulating factor; $\mathrm{k}_{2}$ is the inhibiting factor.

The amount of the antigen $\varepsilon(\mathrm{k})$ is regarded as the error, the total stimulus $\mathrm{S}(\mathrm{k})$ which the $\mathrm{B}$ cells receive is regarded as the control amount $\mathrm{u}(\mathrm{k})$, and the immune control algorithm is deduced from the formula (4), (5), (6):

$$
\mathrm{u}(\mathrm{k})=\mathrm{K}(1-\eta \mathrm{f}(\mathrm{u}(\mathrm{k}), \quad \Delta \mathrm{u}(\mathrm{k})) \quad e(k)
$$

In the above formula, $K=k_{1}$ is the reaction rate of representation control ; $\eta=\frac{k_{1}}{k_{2}}$ is the stable effect of the representation control ; $\mathrm{f}(\cdot)$ is the selected nonlinear function, the ability of inhibiting stimulation of representation cell.

\subsection{The Fuzzy Immune PD Control Principle}

The immune control algorithm which is deduced by the principle of immune feedback [11] is actually a nonlinear $\mathrm{P}$ control algorithm, and its proportion coefficient changes over the control output. Therefore, we can use this algorithm to adjust the $\mathrm{P}$ parameters of PD controller to increase the adaptive ability for the system.

The most difficulty to determine is the nonlinear function $\mathrm{f}(\cdot)$ in the immune control. Considering that the fuzzy control belongs to the nonlinear control, and it is proven to be the universal function approximation method[12], we can select the fuzzy control method to construct the nonlinear function approximation $\mathrm{f}(\cdot)$, see Figure 3-1.

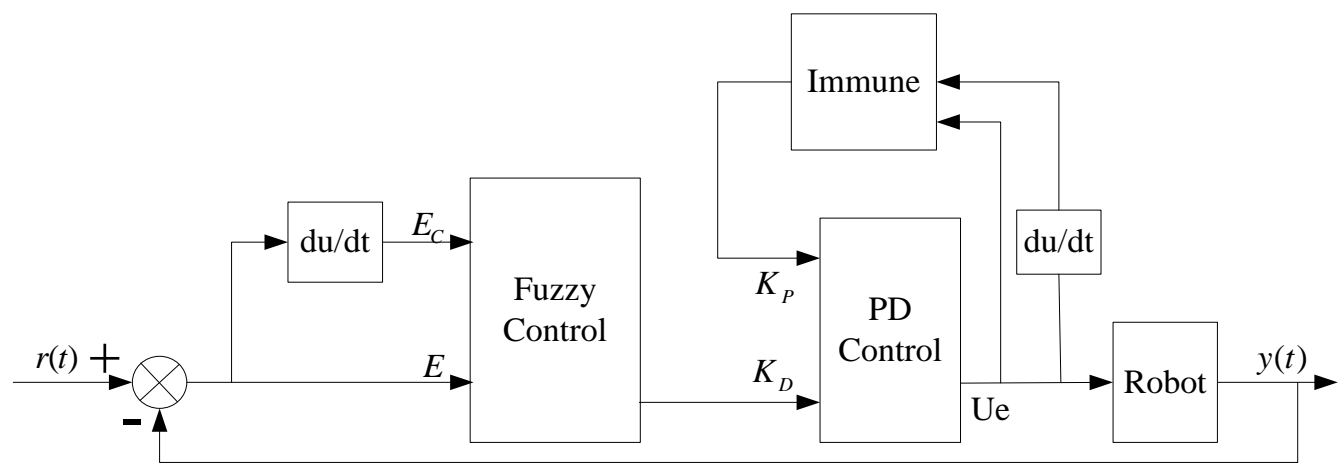

Figure 3-1. The Structure Figure of Fuzzy Immune PD Control 
The system error signal $\mathrm{E}$ and error change rate Ec as the fuzzy controller input, after fuzzy reasoning and decision-making, updating KD values, namely: to get the PD controller output $\mathrm{Ue}$, Ue and its rate of change as an immune regulator input. After adjusting the immune aspects, the resulting variation $\mathrm{KP}$, and then gave $\mathrm{PD}$ controller to update the KP, this closedloop continuous optimization Ue to control the robot.

Using the fuzzy reasoning logic approaching the nonlinear function $f$, the fuzzy input variable is $\Delta \mathrm{u}$, and the fuzzy output variable is $\mathrm{f}$ [13]. Assuming that each input variable is blurred by two fuzzy sets, "positive" (P) and "negative" (N), respectively, the output variables are blurred by three fuzzy sets, "positive" (P), "zero"(Z) and "negative" $(\mathrm{N})$, and the membership functions of $\mathrm{u}, \Delta \mathrm{u}$ and f, respectively, are shown in Figures 3-2, 3-3, 3-4 (the horizontal axis indicates the variables, the vertical axis indicates the membership function MF).
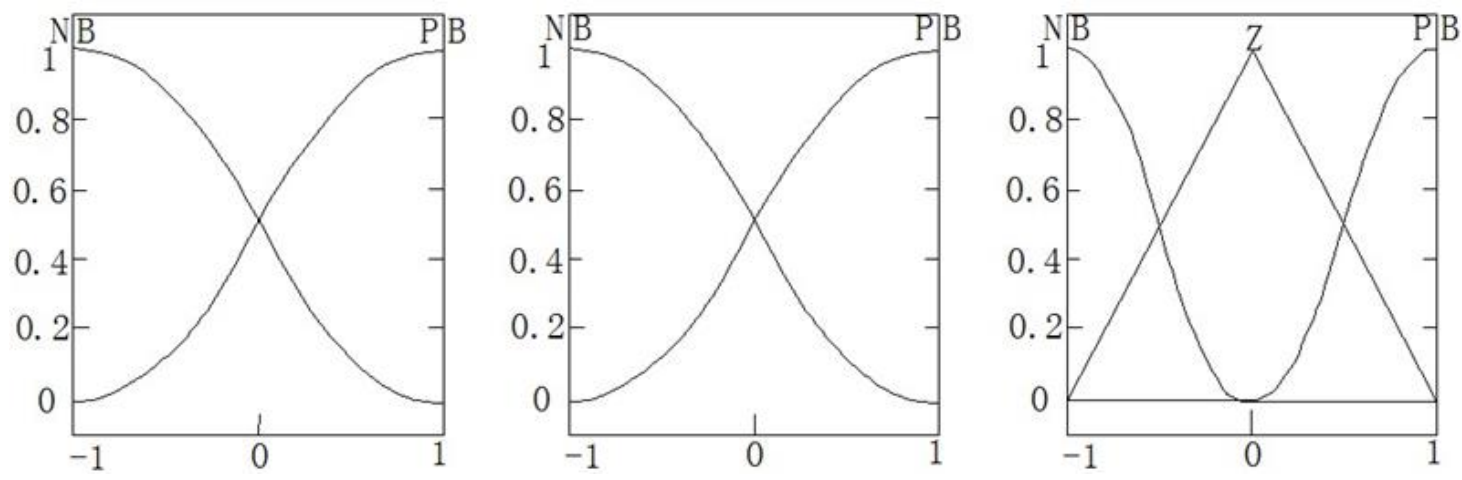

\section{Figure 3-2. The Membership Function of u Figure3-3 The membership function of $\Delta u$ Figure3-4 The Membership Function of $f$}

Using Mamdani fuzzy reasoning [14], the Zadeh fuzzy logic and operation [15] and the common mom anti-fuzzy method [16] get the rules of the variable $\mathrm{u}, \Delta \mathrm{u}$ and $\mathrm{f}$, and they are shown as follows:

(1) If $\mathrm{u}$ is $\mathrm{P}$ and $\Delta \mathrm{u}$ is $\mathrm{P}$ then $\mathrm{f}(\mathrm{u}, \Delta \mathrm{u})$ is $\mathrm{N}$;

(2) If $\mathrm{u}$ is $\mathrm{P}$ and $\Delta \mathrm{u}$ is $\mathrm{N}$ then $\mathrm{f}(\mathrm{u}, \Delta \mathrm{u})$ is $\mathrm{Z}$;

(3) If $\mathrm{u}$ is $\mathrm{N}$ and $\Delta \mathrm{u}$ is $\mathrm{P}$ then $\mathrm{f}(\mathrm{u}, \Delta \mathrm{u})$ is $\mathrm{Z}$;

(4) If $\mathrm{u}$ is $\mathrm{N}$ and $\Delta \mathrm{u}$ is $\mathrm{N}$ then $\mathrm{f}(\mathrm{u}, \Delta \mathrm{u})$ is $\mathrm{P}$;

It can be seen in formula (7) that it is a nonlinear $\mathrm{P}$ controller [17]; the proportion coefficient is $\mathrm{k}_{\mathrm{p} 1}=\mathrm{K}(1-\eta \mathrm{f}(\mathrm{u}(\mathrm{k}), \Delta \mathrm{u}(\mathrm{k}))$, and it changes over the controller output, among them, and the $\mathrm{K}$ is the gain. The immune PD controller output is:

$$
\left.\mathrm{u}(\mathrm{k})=\mathrm{k}_{\mathrm{p} 1}\left((\mathrm{e}(\mathrm{k})-\mathrm{e}(\mathrm{k}-1))+k_{d}(\mathrm{e}(\mathrm{k})-2 \mathrm{e}(\mathrm{k}-2)+\mathrm{e}(\mathrm{k}-2))\right)\right)
$$

\section{The Simulation Experiment of Fuzzy Immune PD Controller}

The simulation experiments are conducted in the simulink environment, and the simulation diagram which has been set up is shown in Figure 4-1. 


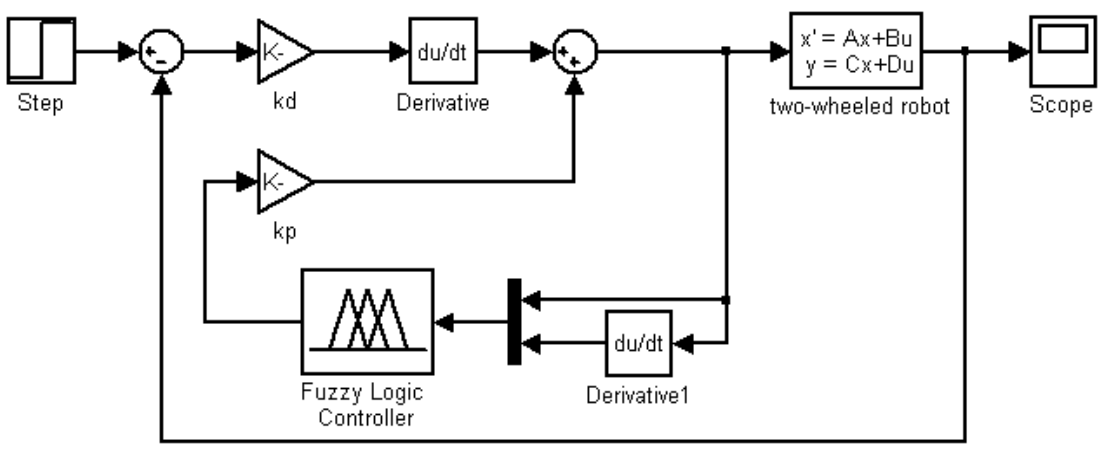

Figure 4-1. The Simulation Diagram of Fuzzy Immune PD Control

The given angle is the balance point angle 0 , and the mathematical model of the two-wheel self-balancing robot is simulated by the conventional PD control, fuzzy PD control and fuzzy immune PD control algorithm, respectively.

The sampling time is $15 \mathrm{~s}$, among them $\mathrm{K}_{1}$ values $0.06, \eta$ values 0.5 . The response curve of step signal in the balance point applied is shown in Figure 4-2. It can be seen the response speed of the fuzzy immune PD is fast from the simulation curve, and it oscillates only once, and the range is small. It converges to zero at about $3.5 \mathrm{~s}$, and it almost has no overshoot. The fuzzy PD which is close to 7s also converges to zero, the overshoot is also smaller, but the overshoot amount is significantly greater than the fuzzy immune PD control. The overshoot amount of PD control curve is the largest, and it reaches 15\%. It converges to zero at about 10 s, so the control effect is inferior to the fuzzy immune PD control and the fuzzy PD control. The response curve of applying interference is shown in Figure 4-3, it can also be seen that the anti-interference curve of fuzzy immune PD control converges to zero quickly; the response speed is fast, and it is faster than the fuzzy PD control and fuzzy control.

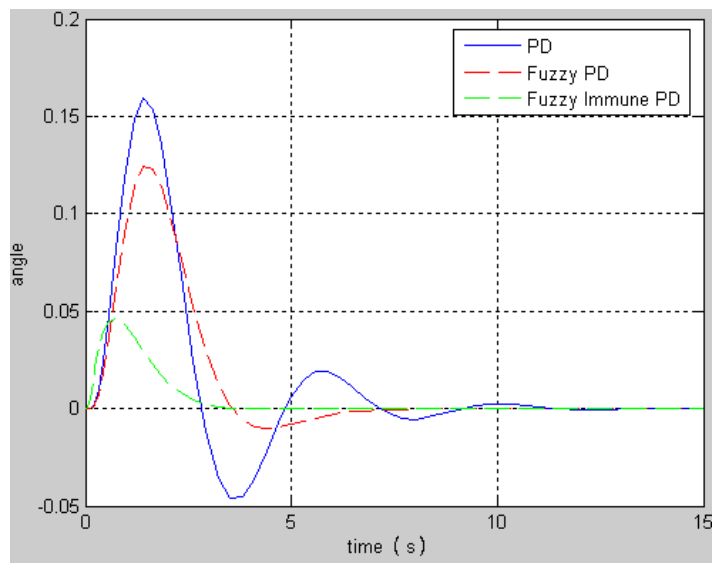

Figure 4-2. The Response Curve Near the Balance Point 


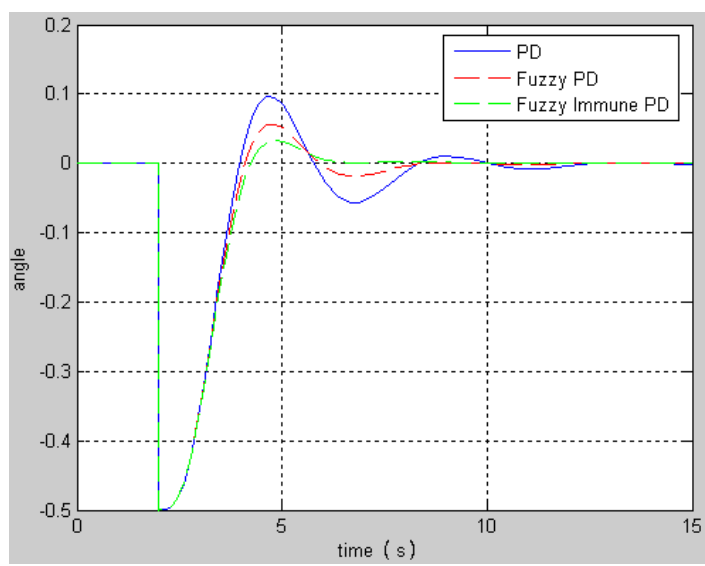

Figure 4-3 The Response Curve After Applying Interference at the Balance Point

In order to compare the traditional PD control, fuzzy PD control and fuzzy immune PD control characteristics of the algorithm, each algorithm control indicators in Table 1.

Table 1 Comparative characteristics of various control algorithms

\begin{tabular}{|c|c|c|c|c|}
\hline Algorithm & Stability & Overshoot & Regulation time & $\begin{array}{c}\text { Oscillation } \\
\text { frequency }\end{array}$ \\
\hline PD & Stable & $16.6 \%$ & $10 \mathrm{~s}$ & 3 \\
\hline Fuzzy PD & Stable & $12.4 \%$ & $7 \mathrm{~s}$ & 1 \\
\hline Fuzzy Immune PD & Stable & $4.9 \%$ & $3.5 \mathrm{~s}$ & 1 \\
\hline
\end{tabular}

\section{The Physics Experiment of the Fuzzy Immune PD Controller}

The physics experiment subject of the control system adopts the self-designed and selfbalancing robot of two rounds, it is shown in Figure 5-1. When the self-balancing robot moves, the gyroscope and accelerometer fusion algorithm are used to measure the angle. The system uses the Bluetooth serial port to communicate and transmit the real-time data information. The data sent by the wireless can be read at any time through the self-developed PC LABVIEW monitoring platform, and a variety of data that the robot walks and operates can be observed. 


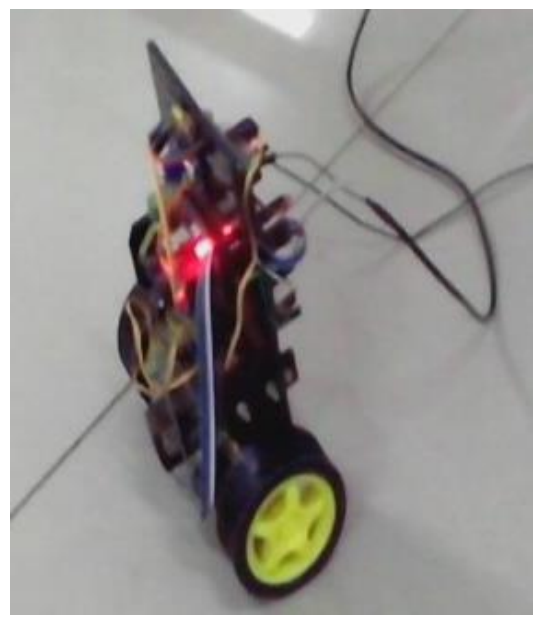

Figure 5-1 The Real Photos of Two-Wheeled Robot

Figure 5-2 is the self-balancing posture test curve near the balance point; the first is the gyro test curve; the second is the accelerometer test curve; the third is the synthetic curve of angle. It can seen that the robot can be stabilized near the center through the analysis of the measured data.

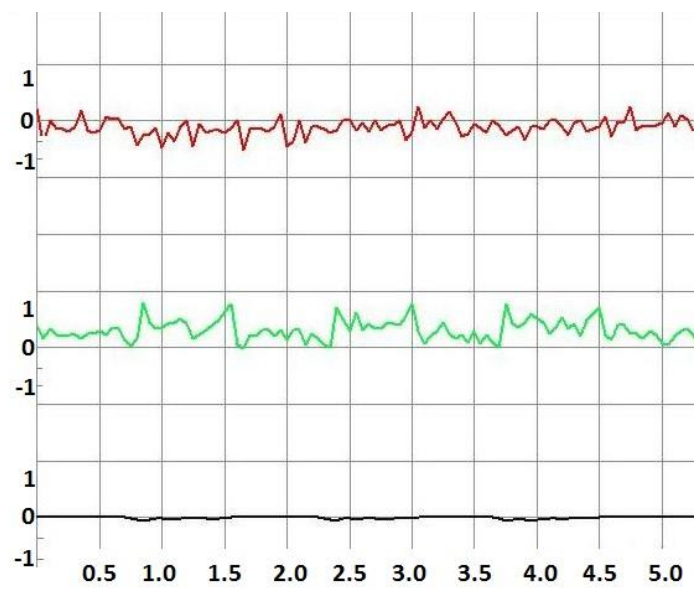

Figure 5-2 The Self-Balancing Test Curve Near the Balance Point

Figure 5-3 is the test curve after applying interference near the balance point. It can be seen that the system can adjust themselves to return to a stable state in 2.5 seconds after suffering a few minor shocks with adding some interference through the analysis of the measured data. 


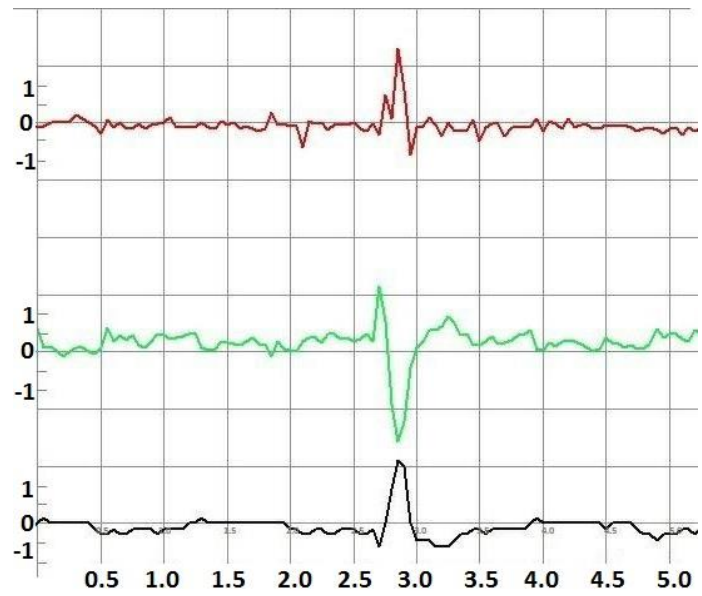

Figure 5-3 The Test Curve After Applying Interference Near the Balance Point

\section{Conclusion}

This paper designs the fuzzy immune PD controller for the two-wheeled and self-balancing robot. The simulation results show that the self-balancing robot system has the characteristics of fast response speed, fewer oscillation times and small overshoot. Based on this method, its control effect is better than the effect of PD and fuzzy PD control, so it has a good application prospect.

Fuzzy Immune PD feedback mechanism to control the use of immune-line adaptive parameters Kp; which implements the optimized PD parameters. Fuzzy Immune PD controller simulation and physical experiments showed that: Fuzzy Immune PD controller inherits the fuzzy PD controller for uncertain mathematical model of the controlled object; simple advantage; also overcome the fuzzy PD control of nonlinear systems with poor mobility shortcomings, to better achieve the two-wheeled self-balancing robot stability control.

Given the biological immune system is a very complex system, which contains a wealth of information processing mechanisms. These information processing mechanism to solve the problem of robot control are of great help. This article exhumed and take advantage of just one small part. Therefore, in this paper, further research work will be genetic, immune algorithm combined with control system, so as to improve the performance of the control system must be able to in dealing with a lot of nonlinear time-varying complex has better performance.

\section{References}

[1] Z. Zhi-qiang, "Two-wheeled self-balance vehicle based on STM32”, Electronic Design Engineering, vol. 9, no. 11, (2007), pp.103-106.

[2] T. Braunl and J. Pan, "Balancing a Two-Wheeled Autonomous Robot", The University of Western Australia School of Mechanical Engineering Final Yinal Year Thesis, (2003).

[3] L. Yang and L. YI-we, "Simulation Research on Self-balanced Two-wheel Vehicle Control Algorithm Based on PID and LQR", MECHANICAL ENGINEERING \& AUTOMATION. (2011), pp.7-10.

[4] H.-S. Juang and K.-Y. Lum, "Design and control of a two-wheel self-balancing robot using the arduino microcontroller board", Control and Automation (ICCA), vol. 1, (2013), pp.634-639.

[5] J. F. Wu and W. Zhan, "Design of fuzzy logic controller for two-wheeled self-balancing robot", Strategic Technology (IFOST), (2011), pp. 1266-1270. 
[6] Y. Qin, Y. Liu, X. Zang and J. Liu, "Balance control of two-wheeled self-balancingmobile robot based on TS fuzzy model”, Strategic Technology (IFOST), vol. 1, (2011), pp. 406-409.

[7] Z. Feng, H. Zheng, L. Zhang, Y. Zhao and W. Huang, "Based on gyroscope and accelerometer signal fusion of attitude Angle measurement", Journal of southwest normal university (natural science edition), (2011), pp. 137-141.

[8] B. Li, J. Yang and H. Ding, "Based on the mechanism of biological immune intelligent scheduling system modeling and simulation", - CIMS computer integrated manufacturing system, (2002), pp. 446-450.

[9] J. K. Liu, “Advanced PID control and MATLAB simulation. Beijing: electronic industry press”, (2003).

[10] Y. Ding, L. Ren, "A novel fuzzy self-tuning immune feedback control system”, Control and Decisionmaking, (2000), pp. 443-450.

[11] Z.-Q. Qi, G.-D. Hu, Z.-H. Yang and F.-E. Zhang, "A novel control algorithm based on immune feedback principle", International Conference, (2002), pp.1089-1092 .

[12] B. Igelnik and Y.-H. Pao, "Stochastic choice of basis functions in adaptive function approximation and the functional-link net", Neural Networks, (1995), pp. 1320-1329.

[13] D. Pei, "Logic based on fuzzy logic and fuzzy reasoning problem ten years of research were reviewed", Journal of Engineering Mathematics, (2004), pp. 249-258.

[14] Z. Yalin, H. Delong and G. Jian, "The Mamdani Fuzzy reasoning algorithm”, Baoji college of liberal arts journal (natural science edition), (2001), pp. 168-173.

[15] W. Pei, "Logic based on fuzzy logic and fuzzy reasoning problem ten years of research were reviewed", Journal of Engineering Mathematics, (2004), pp. 249-258.

[16] H. Chen, "Research of fuzzy method", Automation Panorama, (2008), pp. 71-73.

[17] P. Obstawski, "Utilization digital PID controller to steering electric machine work", [J], Przeglad Elektrotechniczny, (2010), pp. 231-234. 